\title{
Acute Cardiac Tamponade after Left Sided Premature Ventricular Contraction Ablation
}

\author{
Zsuzsanna Kis, Szili Török Tamás, Kovács István \\ Department of Cardiology, Thoraxcenter, Clinical Electrophysiology, Erasmus, Rotterdam, The Netherlands
}

\section{ABSTRACT}

Introduction: Premature ventricular complex (PVC) is the most common cardiac arrhythmia, which in some circumstances can lead to syncope, arrhythmia-induced cardiomyopathy and sudden death. In idiopathic PVCs, the first choice of treatment is radio-frequency ablation. Identification of the substrate location makes it possible to determine the ablation site, leading to an increased success rate. Complications are related mainly to the ablation technique, peri-procedural anticoagulation therapy, and the access site. Pericardial tamponade is a rare complication. A case in which the ablation procedure of idiopathic PVCs, complicated by cardiac tamponade, is presented in this paper.

Case presentation: A 56-year-old female presented with symptomatic premature ventricular contractions. She had frequent palpitations, dyspnea, and exercise intolerance for ten years. Holter-monitoring demonstrated a total burden of 30,549 PVCs with monomorphic morphology, and with both bigeminal and trigeminal patterns. Surface ECG suggested a left-sided, left aortic cusp localization of the PVC, with a possible epicardial origin. Three-dimensional mapping was performed including the RVOT (right ventricular outflow tract) region, aorta, and coronary sinus. The ablation clinical status suggested a cardiac tamponade, which was confirmed by echocardiography. Radioscopy-controlled pericardial puncture was performed with the extraction of $300 \mathrm{ml}$ of blood. Following this maneuver, the general status of the patient improved. During follow-up checks after twenty-four hours, Holter-monitoring recorded 5000 PVCs with a significant improvement in the clinical status of the patient.

Discussions: Pericardial tamponade after radio-frequency ablation is a rare complication. The risk of tamponade in a right chamber perforation is more dangerous in patients on anticoagulation therapy or with pulmonary hypertension. In order to prevent this side effect complication of the interventional procedures, certain safety maneuvers should be followed, including the use of irrigated-type catheters, or when possible, contact force catheters, ensuring invasive arterial blood pressure monitoring during intervention, and after heparin administration and the determination of ACT every twenty minutes. Transthoracic echocardiograph examination and pericardial puncture set should be readily available in the electrophysiology laboratory.

Keywords: PVCs, ablation, tamponade

\section{ARTICLE HISTORY}

Received: 25 April, 2016

Accepted: 28 May, 2016

\section{CORRESPONDENCE}

Tamás Szili Török

Department of Cardiology,

Thoraxcenter, Clinical

Electrophysiology, Erasmus MC

Postbus 2040, S Gravendijkwal 230,

Kamer BD416, 3000, Rotterdam, The

Netherlands

Tel: +31 107033991

Fax: +31 107034420

E-mail: t.szilitorok@erasmusmc.nl 


\section{INTRODUCTION}

Premature ventricular complex (PVC) is the most common cardiac arrhythmia and is observed in $25 \%$ of patients performing exercise tests. ${ }^{1}$ Under certain circumstances, PVCs may initiate ventricular tachycardia (VT), polymorphic VT or ventricular fibrillation, all of which can be the cause of sudden cardiac death. ${ }^{2}$ Catheter ablation, in cases of recurrent VT and monomorphic focal PVCs, is the method of choice for the management of arrhythmia. ${ }^{3}$ The ablation success rate of VT and PVC is between 60 to $90 \%$, with low complication rates. The indication of ablation therapy is based on the patient's symptoms and life quality influences, surface ECG, Holter-monitoring and the location of PVCs. Important signs which predict the location of PVCs are not readily detected by surface ECG, and an electrophysiological study and 3D electro-anatomical mapping is necessary to identify the exact substrate of the arrhythmia. The identification of the substrate location makes it possible to determine the ablation site, and once determined, this will increase the success rate. ${ }^{1}$ Complex electrophysiological equipment is required to perform these procedures, including electro-anatomical mapping systems and irrigated-type ablation catheters. Simultaneous mapping of anatomical regions, such as RVOT, LVOT, aorta, and if possible, the coronary sinus, should be performed to identify the origin of the arrhythmia. Because of the anatomic proximity and continuity of the outflow tracts, the best location to map or to ablate an arrhythmia in the RVOT is often from the supra- or sub-valvular LVOT or vice versa. ${ }^{4}$ Complications of the radiofrequency ablation are related mainly to the ablation technique, and periprocedural anticoagulation therapy. ${ }^{5}$ Pericardial tamponade, or pericardial effusion, is a rare complication which can be related to trans-septal puncture, inappropriate ablation on thin myocardial areas such as the RVOT or the left atrium posterior wall. The occurrence rate of cardiac tamponade at different PVC locations is similar and overall it is the highest with epicardial PVCs (4.2\%). ${ }^{6,7}$ Standard indications associated with a high success rate are: patients undergoing ablation for PVCs with typical indications such as frequent palpitation lasting longer than one year, monomorphic PVC, outflow tract location, $>5000$ PVC/24 hours on Holter-monitoring, without structural heart diseases or cardiac ischaemic history.

A case involving the ablation procedure of idiopathic PVCs complicated with cardiac tamponade is presented.

\section{CASE PRESENTATION}

A 56-year-old female was referred with symptomatic premature ventricular contractions (PVCs). The patient had been experiencing frequent palpitations, dyspnea and exercise intolerance for about ten years. Her medical history included hypertension, with no sudden death occurring in her family. Antiarrhythmic drugs including beta-blockers and amiodarone had been prescribed by her general practitioner. Changes in lifestyle failed to resolve her symptoms. Twenty-four hour Holter-monitoring demonstrated a total burden of 30,549 PVCs with monomorphic morphology, and with bigeminal and trigeminal patterns. Surface ECG suggested a left-sided, left aortic cusp localization of PVC with possible epicardial origin (Figure 1).

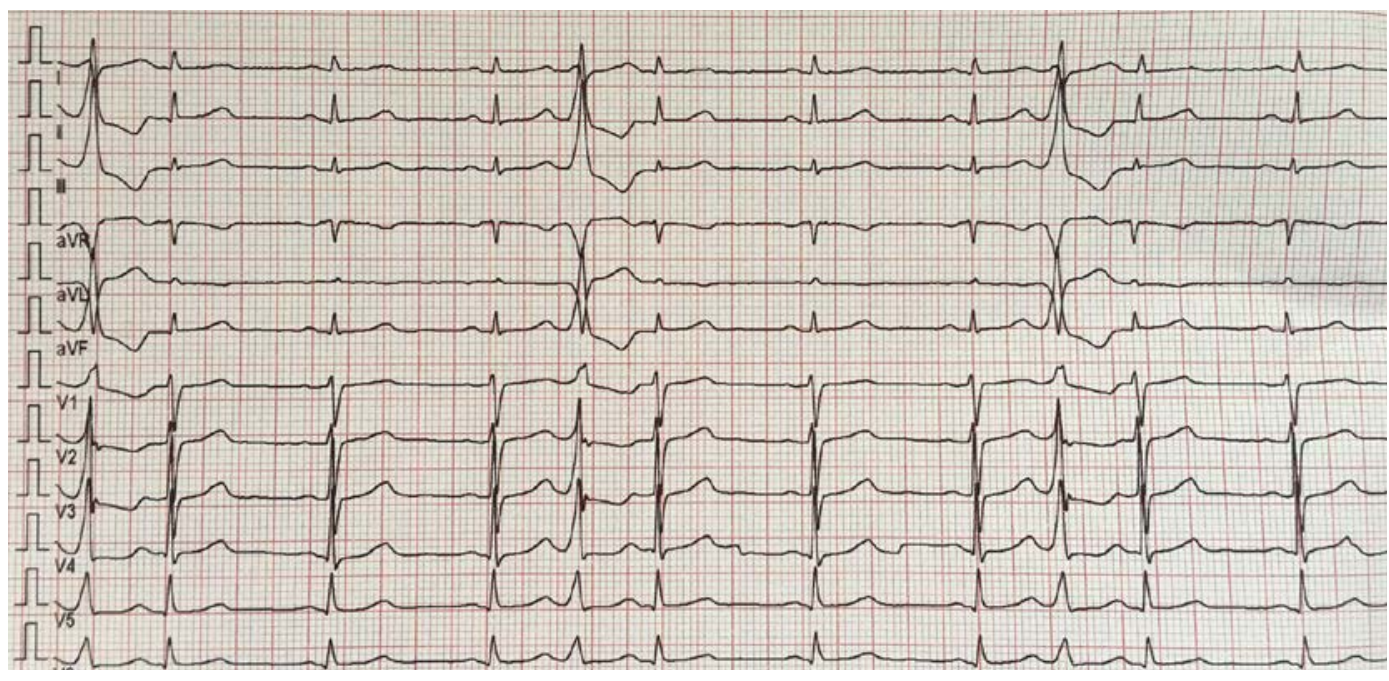

FIGURE 1. 12-lead ECG demonstrates monomorphic premature ventricular contraction (PVC), left aortic cuspe localisation of PVC with possible epicardial origin 


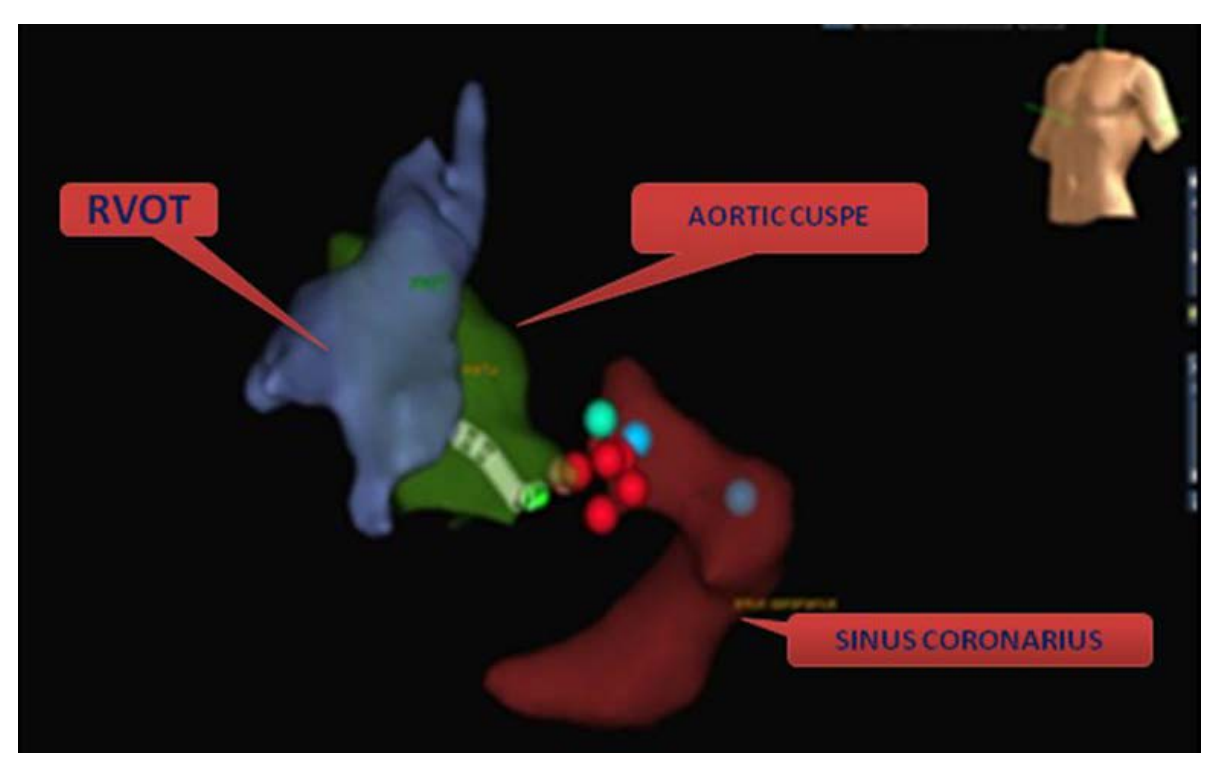

FIGURE 2. Electroanatomical map of the right ventricular outflow tract (RVOT), coronary sinus, aortic cuspe and the ablation site (red spots).

The two-dimensional echocardiography examination revealed a maintained left ventricular systolic function, with an estimated ejection fraction of $50 \%$, without wall motion or valvular abnormalities. The coronary angiography was negative.

Due to the increased symptomatic burden of PVCs, as well as no response to medication therapy, she was considered for radiofrequency ( $\mathrm{RF}$ ) ablation.

Electrophysiology (EP) procedures were performed in the EP lab. After obtaining informed consent, 8 French sheaths were inserted in the right femoral veins and artery. From right radial access site, a 5F left coronary diagnostic catheter was inserted in order to prevent a left main lesion, because an aortic cusp ablation site was predicted.
A 3D map was obtained using a 7 French, Cool Path ${ }^{\mathrm{TM}} \mathrm{Ab}-$ lation, Bi-directional Irrigated $4 \mathrm{~mm}$ Tip Thermocouple Catheter (St. Jude Medical) and the EnSiteNavX Navigation system (St. Jude Medical). The patient had multiple unifocal PVCs at baseline. Three-dimensional mapping was performed including the RVOT region, aorta and coronary sinus (Figure 2).

The earliest activation region was located in the left ventricular sub-valvular site. Pacing from the same site produced a perfect match (Figure 3). At the beginning of procedure, heparin and saline was used to flush-out the sheets, and before the ablation procedure 5000 UI heparin was given intravenously. After twenty minutes of heparin administration an activation clotting time (ACT) of 250

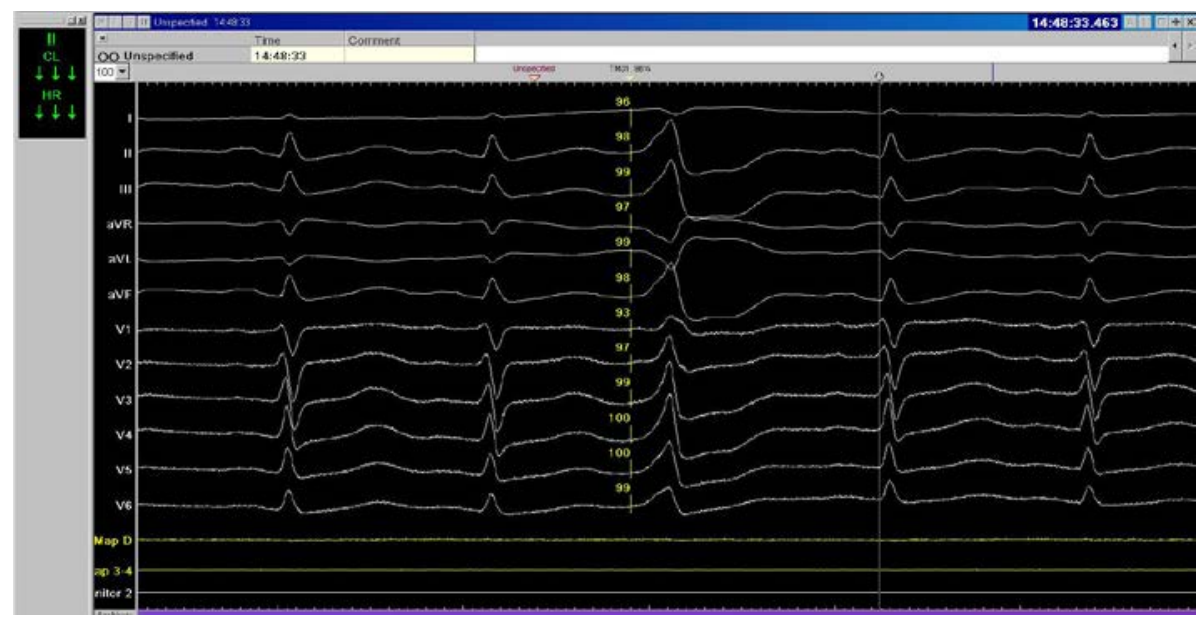

FIGURE 3. Intracavitary electrocardiogram shows $>98 \%$ superposability of the signs registered on ablation site (during pacing) and the clinical PVC 


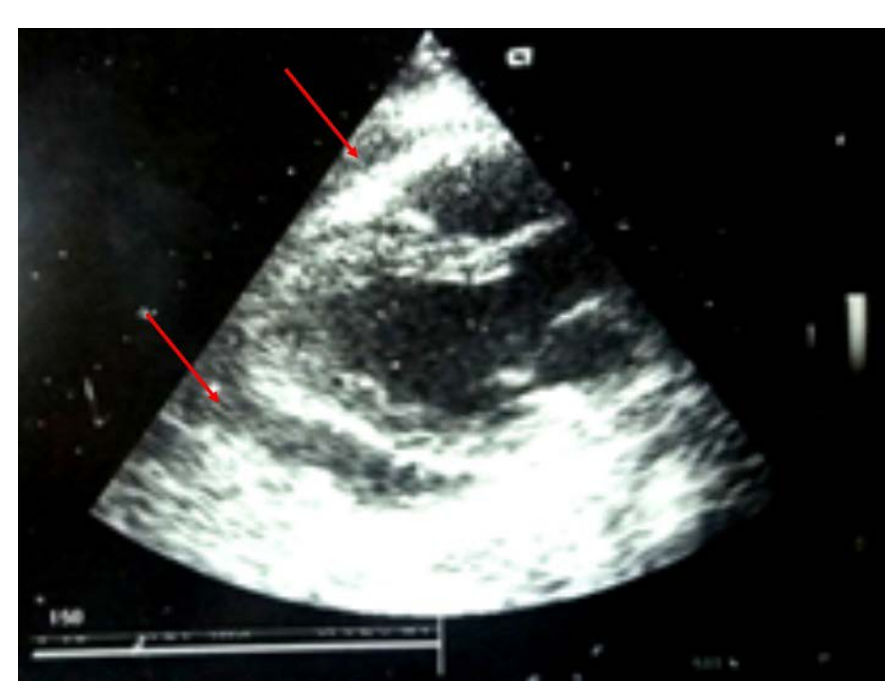

FIGURE 4. Transthoracic echocardiography shows significant fluid accumulation in the pericardial sac (red arrow).

seconds was recorded. Multiple RF lesions were produced at that particular area (in the left ventricle in the aortic subvalvular region) resulting in termination of the PVCs. After Izuprel administration, isolated PVCs appeared with different characteristics compared to the clinical PVC. On moving the ablation catheter in the sub-valvular region, the patient suddenly presented with severe dyspnea, chest pain associated with hemodynamic decompensation. Urgent transthoracic echocardiography showed a cardiac tamponade with significant fluid accumulation in the pericardial sac (Figure 4). Radioscopy controlled pericardial puncture was performed with the extraction of $300 \mathrm{ml}$ of blood. Immediately Protamin was administered. Following this, there was an improvement in the general status of the patient. Control echocardiography after one, six and twenty-four hours did not show any pericardial fluid accumulation (Figure 5).

On follow-up after four weeks, echocardiography indicated no accumulation of pericardial fluid. Holter-monitoring recorded 5000 PVCs in twenty-four hours, and this was monomorphic in nature and of a different pattern in comparison to that previously recorded. The clinical status of the patient had improved significantly.

The patient gave informed consent allowing publication of patient data, and the institution where the procedure was performed approved the publication of the case.

\section{DISCUSSIONS}

Pericardial tamponade after radio-frequency ablation is a rare complication, especially in cases where irrigated-type and contact force ablation catheters are used. ${ }^{2,4}$ If mechanical perforation occurs, pericardiocentesis is usually suf-

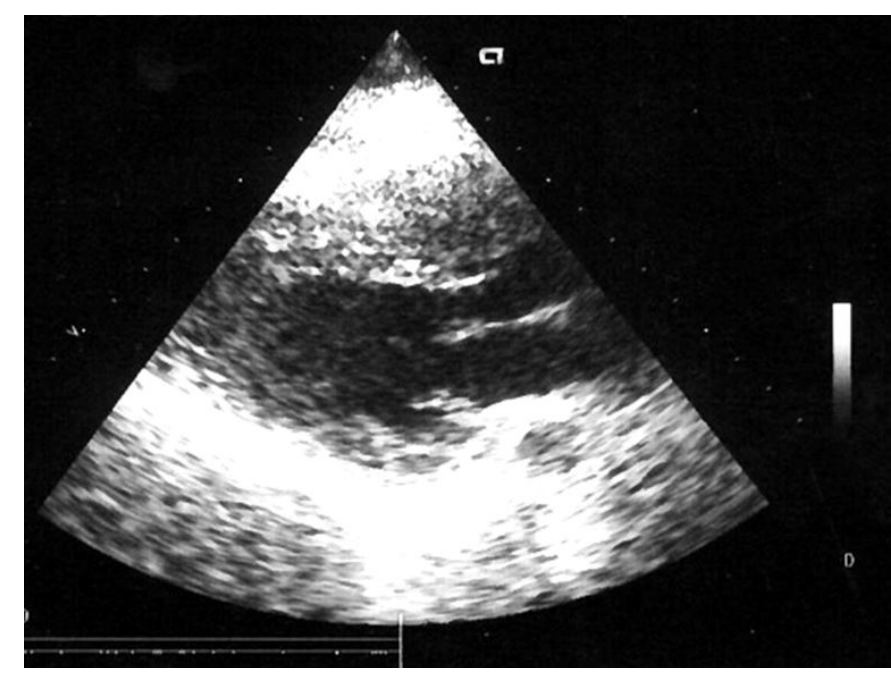

FIGURE 5. Control echocardiography after $300 \mathrm{ml}$ blood extraction, without pericardial fluid.

ficient to stop the bleeding. When the tamponade is related to the ablation, i.e. the burned myocardium lesion, occasionally it is necessary to initiate urgent surgical hemostasis. ${ }^{6}$ The prevalence of pericardial effusion is influenced by the location of the PVC/ablation site. RVOT and left atrial ablations present a higher percentage of tamponade because of anatomical factors. Perforation of the RV may not result in clinical deterioration. The risk of tamponade in a right chamber perforation is more dangerous in patients on anticoagulation therapy or with pulmonary hypertension. ${ }^{6}$ The RV transmural thickness is $4 \mathrm{~mm}$ and is associated with a lower incidence of iatrogenic perforation with electrophysiology catheters. Perforation of the thicker wall (circa $10 \mathrm{~mm}$ ) at the LV site, is less frequent. However, the higher left ventricular pressure increases the tamponade risk especially in anticoagulated patients. ${ }^{6}$

In order to prevent complications associated with interventional procedures, certain safety maneuvers should be strictly followed, such as using irrigated-type catheters, contact force catheters when possible, and ensuring invasive arterial blood pressure monitoring during the intervention procedure, accompanied by the determination of ACT after heparin administration, every twenty minutes. The ablation procedure should be started when the ablation site stimulation and clinical PVC are 100\% matched. Both transthoracic echocardiography examination and pericardial puncture set should be easily available in the EP laboratory.

\section{CONFLICT OF INTEREST}

Nothing to declare. 


\section{REFERENCES}

1. Bohnen M, Stevenson WG, Tedrow UB, et al. Incidence and predictors of major complications from contemporary catheter ablation to treat cardiac arrhythmias. Heart Rhythm. 2011;8(11):1661-1666. DOI: 10.1016/j.hrthm.2011.05.017

2. MüssigbrodtA, GrothoffM, DinovB, etal. IrrigatedTipCatheters for Radiofrequency Ablation in Ventricular Tachycardia. BioMed Research International. 2015(2015); Article ID 389294 , 6 pages. DOI: http://dx.doi.org/10.1155/2015/389294

3. Aliot EM, Stevenson WG, Almendral-Garrote JM, et al. EHRA/ HRS Expert Consensus on CatheterAblation of Ventricular Arrhythmias. Heart Rhythm. 2009;6(6):886-933. DOI: 10.1016/j.hrthm.2009.04.030.

4. Shah D, Lambert H, Langenkamp A, et al. Catheter tip force required for mechanical perforation of porcine cardiac chambers. Europace. 2011;13(2):277-283. DOI: 10.1093/ europace/euq403.

5. Tokuda M, Kojodjojo P, Epstein LM, et al. Outcomes of Cardiac Perforation Complicating Catheter Ablation of Ventricular Arrhythmias. Circ Arrhythm Electrophysiol. 2011;4(5):660666. DOI: 10.1161/CIRCEP.111.963413.

6. Holmes DR Jr, Nishimura R, Fountain R, Turi ZG. Iatrogenic Pericardial Effusion and Tamponade in the Percutaneous Intracardiac Intervention Era. JACC Cardiovasc Interv. 2009;2(8):705-717. DOI: 10.1016/j.jcin.2009.04.019.

7. Latchamsetty R, Yokokawa M, Morady F, et al. Multicenter Outcomes for Catheter Ablation of Idiopathic Premature Ventricular Complexes. JACCCEP. 2015;1(3):116-123. DOI: 10.1016/j.jacep.2015.04.005 